\title{
Tennis is a game of strong and resilient confidence
}

Janet Young (Victoria University, Australia)

ITF Coaching and Sport Science Review 2015; 65 (23): 3 - 5

\section{ABSTRACT}

This paper reviews confidence' and why it is important to players. Strategies for coaches to guide players in developing, maintaining and regaining (if required) confidence are highlighted.
Key words: confidence, coach, mental, belief

Received: 15 January 2015

Accepted: 14 March 2015

Corresponding author:

Janet Young

Email:janet_young7@yahoo.com.au

\section{INTRODUCTION}

It is not uncommon for coaches to tell their players to be confident and/or to play confidently. Such advice is sound but it often leaves players at a loss to know how to be confident, how to maintain it during a match, tournament event or season and, if required, how to regain it if lost. Players generally know when they feel confident - they are positive, focused, goaldirected and self-assured, hit the ball cleanly and go for their shots, thrive on the pressure of training and competition and have no doubt as to their ability to perform well. Awareness of being confident may not be sufficient with some players in need of a coach's help to gain a fuller understanding of the 'what', 'why' and 'how' of this much desirable and admired attribute. To address these aspects let us examine confidence in more detail and see what role a coach can play in guiding players to develop and nurture confidence.

\section{WHAT IS CONFIDENCE?}

Interestingly, there are numerous definitions of confidence to be found in sport literature. A popular one proposed by Weinberg and Gould is "The belief or degree of certainty that individuals possess about their ability to be successful in sport" (p. 322). More simply conceptualised by Rotella (2004), confidence is "thinking about the things you want to happen" (p.37). In this context, players who are confident think about what they want to happen on the court in contrast to players who lack confidence thinking about the things they do not want to happen on the court.

In terms of the nature of confidence, a number of key features are listed in Table 1.

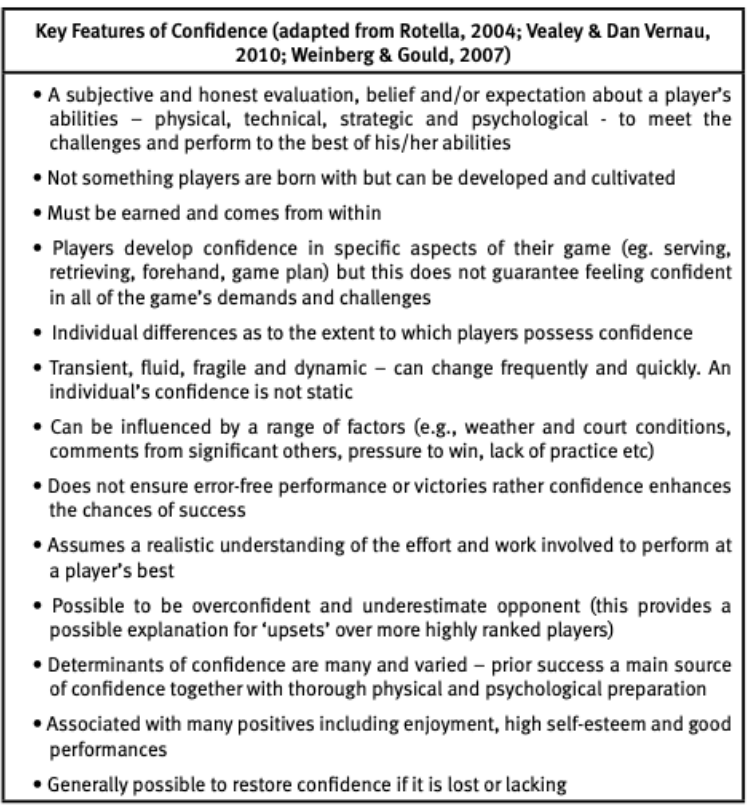

Table 1. Key Features of Confidence.

As noted in Table 1, confidence is not what a player hopes or wants to do but rather is a realistic and positive anticipation of what a player expects will happen. Confidence reflects a player's innermost thoughts about what he/she is capable of doing and fully appreciating the work and effort required to perform well. Confidence does not guarantee success but certainly increases the chances of it happening. It is however noted that players can possess too much confidence and be 'over-confident' as to the outcome of matches. Sometimes in these matches opponents are underestimated and 'upset' results can occur.

Considerable time and effort is generally required when it comes to developing confidence. Genuine and resilient confidence is earned after many hours, days, weeks and years of dedicated work and commitment to the game. There is no magic wand! However it is thought that the dedicated work and 
effort are well worthwhile because confidence brings its own rewards including:

- Assisting a player to develop a 'winning' (versus 'not losing') match strategy

- Allowing a player to focus on relevant cues during a match and not be distracted by his/her own self-doubts

- Facilitating good decision-making because a player is thinking positively and constructively

- Arousing positive emotions including feeling relaxed, competent and in control

- Helping a player 'to dig deep' at critical times during matches

- Enabling a player to give his/her best efforts and enjoy his/her tennis

It is also noted from Table 1 that confidence is generally a fragile attribute. For many players confidence can be quickly and easily lost. A range of factors can contribute to this including a player's own self-doubts, an unexpected loss or a 'string of losses' and negative comments from significant others (including coach, parent and other players). Warning signs of a loss of confidence can include a lack of enthusiasm and enjoyment in playing and training, serving double faults in pressure situations and an inability to execute certain shots or play as would normally be the case for that player. To understand how best to maintain confidence, and regain if required, let us further explore the ways players might build confidence.

\section{How to Build Confidence}

It is thought that the main ways a player can build confidence include:

a) Persistent, deliberate practice and training of physical and psychological skills

b)Receiving constructive and positive feedback, encouragement and guidance from a support team, including coach, parents and friends

c) Successful performance accomplishments - be it wins, good performances and/or making progress in skill/game development and achieving goals or targets.

Recently Vealey and Vernau (2010) suggest that systematic physical preparation allows players to trust themselves in executing their skills during the pressure of competition. In a similar vein these authors propose that systematic psychological preparation facilitates sound decision making and allows players to respond appropriately to competitive pressure, distractions and setbacks. To this end players may engage in positive self-talk and imagery for example. Coaches and other support persons are deemed to be important in setting the right 'tone' and culture around a player. An environment that reinforces the abilities, capabilities and potential of players is conducive to players feeling confident about themselves and their game. Finally, nothing breeds confidence better than 'success'. Vealy and Vernau highlight the confidence that can be derived from solid match performances (not only wins but also losses where a player plays to his/her ability) and reaching targets or goals for developing their skills.

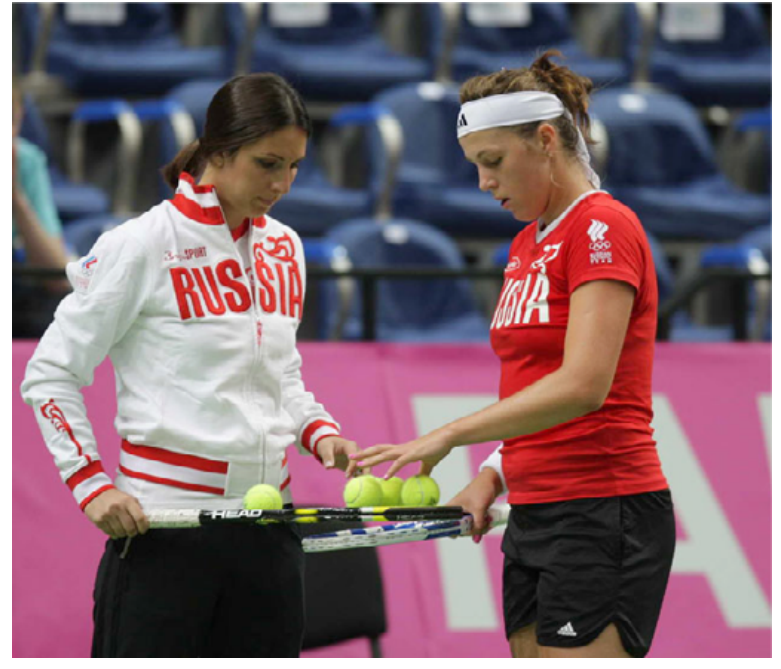

ROLE OF THE COACH

It has been identified that coaches can help players develop, maintain and regain (if required) confidence (e.g., Vealey \& Vernau, 2010; Weinberg \& Gould, 2007). So what can coaches do? Some suggestions follow for consideration. Although these are listed in separate categories (for developing, maintaining and restoring a player's confidence) there is considerable overlap with some suggested strategies applicable at all times when guiding a player to achieve his/her full potential.

What coaches can do to help a player develop confidence

- Encourage and assist a player to set realistic, challenging and achievable goals. Then support a player to work towards achieving these goals, regularly giving positive and constructive feedback as progress and targets are achieved

- Focus on a player's skill development and bring in, or refer players to, fitness specialists or sport psychologists to help fully develop physical and psychological skills if these areas are considered outside a coach's area of training and expertise

- Set up regular match play scenarios that simulate pressure situations where players can test their skills, assess the progress they are making, identify areas for additional work and achieve some success

- Lead by example. Assume a positive disposition and enjoy one's coaching in accord with high standards of ethical conduct - Be mindful of what you say to players. Words need be chosen wisely and should help to make players feel good about themselves and their tennis

- Select players (e.g., Roger Federer, Rafael Nadal and Serena Williams) as illustrations of ideal role models for players to observe and emulate

- Encourage players to recall, and record, their 'best performances' and identify what things they were doing, feeling or experiencing prior to and during such matches. Suggest a player use this information to form a 'template' to follow to play well on a regular basis

- Offer extra coaching, training and practice sessions to allow players to feel that their shots, play and fitness and other skills are at levels players are happy with

- Allow players to learn, make mistakes and take responsibility for their decision making. Facilitate a learning environment where players are consulted and, indeed, are empowered to take control over what happens out on the court

- Together with a player, put together a support team (e.g, trainer) that a player feels believes in him/her 
What coaches can do to help a player maintain confidence - Provide a positive, consistent, fun and supportive coaching environment

- Adopt an 'open-door' policy where players feel comfortable to discuss any issues of concern. Assure players that any issues will be addressed in a confidential, non-judgmental and prompt manner

- Discuss with players their template (as above) for best performances. Encourage players to 'replay' in their minds those times when they played at their best and picture themselves repeating such occasions

- Offer extra match play, training, practice and coaching to 'fix' or improve any aspect of a player's game that is causing concern

- Refocus players' attention on the '3 Ps' (i.e., preparation, practice and planning for matches)

- When watching players compete, attend to your own body language and refrain from showing despair, concern or disappointment. Provide constructive feedback after matches that focus on what players did well and also areas for on-going improvement. In these discussions continue to stress development, addressing challenges and improvement (versus match wins or losses)

- Regularly consult with players as to the progress they are making to meet goals and targets. Seek suggestions from players if goals and targets are behind schedule and then work with them to revise or update their coaching program

What coaches can do to help a player regain confidence

- Remove the distraction of 'winning' for players and refocus on 'improvement' and 'fulfillment of potential' as measures of success

- Reassess with players their goals and targets to ensure they are not overly demanding but still challenging and achievable

- Review with players any video clips, newspaper articles or other material that highlight a player's achievements or good performances. Use these reminders to rekindle players' enthusiasm for the game

- Remind players that confidence can, in most cases, be restored and rebuilt. Ask players for their own solutions to any pressing issues or concerns and provide assurances that you will provide support (including coaching and training) where possible

- Keep a balanced perspective and remind players to do so themselves. Lead by example by not panicking or being judgmental but rather be realistic that changes/fluctuations in confidence are to be expected

- Facilitate a 'forward looking' approach where learning and perfecting the game is fun but very challenging

\section{CONCLUSION}

A player's confidence is continually tested in tennis. Points are invariably lost in any match, easy shots can be missed and players' 'winning streaks' always end. Defeats are inevitable even for champions. Accordingly, players need to work at their confidence. It is not 'a given' for anyone and requires cultivating, nurturing and sometimes restoring. This article suggests that the goal for players is to have strong, resilient and realistic confidence and, to this end, coaches have a very significant role to play. They can guide and support players to find solutions to a fear of playing/losing, nervous doublefaulting and a loss of enjoyment in playing that are often symptomatic of a lack of confidence. Tennis is a game of confidence and, although confidence comes from within a player, coaches provide a vital clue as to why some players find the answers to see their shots consistently go to their target. That's confidence! But an individual approach by a coach is vital. No two players are the same and a holistic, caring, empathetic and supporting approach is required. Confidence must be earned just as Roger Federer and Serena Williams have done in their pursuit of establishing a tennis legacy.

\section{REFERENCES}

Rotella, R. (2004). The golfer's mind. New York, NY: Fine Press.

Vealy, R.S., \& Vernau, D. (2010). Confidence. In S. Hanrahan \& M. Anderson (Eds.), Routledge handbook of applied sport psychology: A comprehensive guide for students and practitioners (pp.5-8-527). New York: Routledge.

Weinberg, R., \& Gould, D. (2007). Foundations of sport and exercise psychology (4th Ed.). Champaign, II: Human Kinetics. .

RECOMMENDED ITF TENNIS ACADEMY CONTENT (CLICK BELOW)

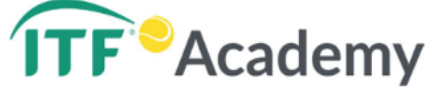

Copyright (c) Janet Young 2015

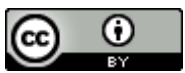

This text is under a Creative Commons BY 4.0 license

You are free to Share - copy and redistribute the material in any medium or format - and Adapt the content - remix, transform, and build upon the material for any purpose, even commercially under the following terms:

Attribution: You must give appropriate credit, provide a link to the license, and indicate if changes were made. You may do so in any reasonable manner, but not in any way that suggests the licensor endorses you or your use.

CC BY 4.0 license terms summary $\quad$ CC BY 4.0 license terms 